\title{
Cancer chemotherapy agent-induced perivenous extravasation injuries
}

\author{
A. Banerjee, T.M. Brotherston, B.G.H. Lamberty and R.C. Campbell \\ Department of Plastic Surgery, Addenbrooke's Hospital, Hills Road, Cambridge, CB2 2QQ, UK.
}

\section{Introduction}

Chemotherapy occupies a well defined role in tumour management and has transformed the prognosis of many haematological neoplasms and solid tumours. Unfortunately, the large majority of chemotherapeutic agents currently in use are toxic to tissues in general and produce tissue necrosis on perivenous extravasation. The sequelae add considerably to the morbidity of an already seriously ill patient, prolonging hospitalization and often necessitating temporary cessation of therapy. The potential for litigation is high, as these are mostly avoidable complications. ${ }^{1}$ Furthermore, this complication is unacceptable if the patient is receiving adjuvant therapy, often as part of a clinical trial.

The need for early surgical attention to these injuries is not widely appreciated, and delay in obtaining surgical advice is common. ${ }^{2}$ Prolonged morbidity results from the consequent delay in definitive management.

This review aims to discuss the pathogenesis, prevention and management of these injuries and attempts to provide useful guide-lines in clinical practice.

\section{Pathogenesis}

Since the first report $^{3}$ of doxorubicin-induced chemical cellulitis, sufficient data has accumulated to allow delineation of a specific type of tissue injury, with a characteristic evolution.

The initiating episode is always painful. The occurrence of pain, or a burning or tingling sensation along the course of the vein being injected should alert one to the possibility of extravasation, even in the absence of confirmatory visual evidence. ${ }^{4}$

Correspondence: A. Banerjee, M.S., F.R.C.S., Department of Plastic Surgery, Wexham Park Hospital, Slough SL2 4HL, UK.

Accepted: 11 August 1986
An initial phase of nonblanching erythema, representing deposition of red doxorubicin crystals in the tissues ${ }^{5}$ is followed sequentially by oedema, brawny induration and full thickness skin necrosis, progressing to ulceration. ${ }^{6}$ The ulcer exhibits a necrotic yellow base, with a surrounding rim of $1-2 \mathrm{~cm}$ of erythema. The edges are rolled and tender. Lack of granulation tissue formation and of peripheral healing by epithelial ingrowth characterize the absence of spontaneous healing. ${ }^{7}$ Sequential histological studies of doxorubicin-induced lesions in rabbits ${ }^{8}$ revealed a necrotizing lesion with a characteristic lack of inflammatory response. A vasospastic mechanism was proposed. Histologically blood vessels are normal in acute lesions. ${ }^{\text {? }}$

Studies of wound healing in doxorubicin-induced lesions in a rat model ${ }^{9}$ showed a predictable dose and concentration dependent skin necrosis with intradermal injections. Further studies on this model ${ }^{10}$ revealed normal myofibroblast ultrastructure suggesting that lack of wound contraction was related to cellular dysfunction, secondary to alterations in nuclear function caused by DNA base binding.

Prolonged tissue retention of doxorubicin is well known, and tissue retention has been demonstrated at up to 5 months after injury. ${ }^{11}$ This has been attributed to lipophilia and to the formation of doxorubicinDNA complexes, the formation of which was first described by Silvestrini. ${ }^{12}$ The nuclear effects of complex formation include intercalation between DNA base pairs and inhibition of DNA-dependent RNA synthesis. ${ }^{13}$

The chronicity and progressive nature of doxorubicin-induced ulcers have been postulated as being due to the liberation of doxorubicin-DNA complexes from dead cells. These complexes are taken up by viable cells by endocytosis, thereby initiating a selfperpetuating recycling process locally. ${ }^{14}$ This provides strong support for early excision of these lesions, confirmed by rat model studies showing that immediate excision of the area of doxorubicin infiltration 
uniformly prevented the development of ulcers. ${ }^{15}$

The possible protective action of the immediate administration of local infusants in preventing or modifying extravasation injuries has been studied in animal models by various authors. While the use of hydrocortisone was suggested on an empirical basis by Reilly et al. ${ }^{17}$ Coleman et al. ${ }^{15}$ demonstrated that it may actually increase the extent of skin necrosis.

Support for the use of a cytoprotective dimethylsulphoxide in treating tissue necrosis has been advocated $^{18,19}$ although this claim is contradicted by other workers. Dorr and Alberts ${ }^{20}$ suggested the use of beta-adrenergic agents but provide no conclusive evidence in favour. Petro et al. ${ }^{21}$ however, using a rabbit model, were unable to find any benefit with the immediate administration of triamcinolone, heparin, sodium bicarbonate, calcium gluconate, magnesium sulphate and $10 \%$ dimethylsulphoxide in doxorubicin extravasation wounds. The role of local infusants in limiting tissue necrosis related to doxorubicin is, therefore, by no means proven and their effects inconsistent.

\section{Prevention}

Attention to preventive measures is essential in view of the increasing frequency of use of intravenous chemotherapy regimes, often by relatively inexperienced personnel. Dilution of the agent in recommended diluent (as obtained from the manufacturers' recommendation sheet) is essential, as lower concentrations cause a lesser extent of tissue necrosis for a given volume of extravasation.

The selection of vein site is important. Veins on the dorsum of the hand are unsatisfactory due to the lack of subcutaneous tissue, and the proximity of extensor tendons and bones and joints to the skin. Veins in the antecubital fossa are in close proximity to neurovascular structures and are better preserved for collection of blood samples. Also, extravasation is difficult to detect in this area. Lower limb veins are best avoided, as their use necessitates restriction of ambulation, as well as being associated with a higher incidence of phlebitis.

The problems of venous access in individuals, especially children, who have already had multiple injections of chemotherapeutic agents with a sclerosing action on veins, is well known. This has encouraged the use of long term central venous and right atrial lines, and the early construction of subcutaneous arteriovenous fistulas to allow reliable repeated venous access with minimal patient discomfort and a decreased risk of extravasation. Radiological confirmation of the siting of central venous catheter tips prior to commencing infusion will avert the risks of chest wall, pleural, pericardial or mediastinal infusion.
Table I Known vesicant agents which cause local necrosis

Actinomycin D
Bleomycin
Daunomycin
Doxorubicin
Mechlorethamine (Nitrogen Mustard)
Mithramycin
Mitomycin
Streptozotocin
Vinblastine
Vincristine

Short term infusions may be given through either scalp vein needles, which are easy to secure as well as retaining some flexibility, or through plastic cannulas, which are preferable to steel needles in view of a decreased extravasation risk..$^{22}$

Limbs with elevated venous pressure caused by such conditions as metastatic compression of major veins must be avoided as there is increased venous permeability and extravasation risk. For the same reason, proximal tourniquets must be removed before injection.

Extremities which have previously been irradiated must be avoided as doxorubicin is known to activate a 'recall phenomenon', a reaction of latent radiation effects in a previously irradiated field. ${ }^{28}$ Interestingly Mamako ${ }^{29}$ has reported exacerbation of a previousl healed area of doxorubicin extravasation after furthe administration of the same agent. Dragon \& Braine $^{30}$ caution the need for using only venous segments proximal to an arteriovenous fistula to avoid potential back diffusion and stasis of high concentrations of irritants leading to cellulitis and tissue necrosis.

The actual technique of venepuncture is important Multiple punctured veins and veins distal to those are not suitable for injections as leakage can occur through holes in the vein wall. Secure fixation of the cannula is essential, but the injection site must be visible at all times. Venous patency must always be tested by a prior saline flush, observing the injection site for obvious swelling.

Serial small volume pushes of diluted drug with constant vigilance and periodic aspiration of blood are preferable to unattended infusions. The use of pressure infusions and infusion pumps is contra-indicated as high infusion pressures can be generated which overcome tissue pressure and increase the risk of extravasation injury. The patient should be advised to report pain, burning, tingling or itching, which suggest perivenous infiltration. ${ }^{4}$ Finally, the drug is followed with a saline flush to clear the tubing and cannula of all residual drug.

The 'Adriamycin flare' reaction ${ }^{31,32,33}$ is reported to occur with $3 \%$ of all doxorubicin infusions. This 
consists of transient erythema, induration, urticaria and pruritus along the course of a vein, proximal to the injection site, which is painless and subsides spontaneously within one hour. This may represent either a local hypersensitivity reaction or represent transient endothelial extravasation, and must not be confused with a true extravasation injury.

\section{Management}

This consists of immediate measures to limit tissue damage and ongoing wound management (Table II).

Ignoffo et al ${ }^{23}$ describe a protocol for the immediate management of extravasations which consists of immediate cessation of injection, and aspiration of $3-5 \mathrm{ml}$ of blood with the needle in place, and local instillation of the recommended antidote (as recommended in their article), and possibly also of steroids. The needle is then removed. Any subcutaneous blebs are aspirated with a 27 gauge needle tuberculin syringe. Warm to hot compresses are applied for 60 minutes to allow vasodilation and improved clearance of residual extravasated drug.

However, the immediate application of ice packs, continued by 20 minute applications 4 times daily for 72 hours is advocated by Larson ${ }^{24}$ in order to inactivate local toxic effects of doxorubicin. The use of ice is also recommended by Barlock et al. ${ }^{25}$ The case for either ice packs or warm compresses, however, remains unproven, as does the role of specific antidotes. Dorr \& Fritz ${ }^{4}$ report the use of sodium thiosulphate as a specific antidote for mechlorethamine extravasations. Zweig \& Kabakow $^{26}$ suggest that the use of $8.4 \%$ sodium bicarbonate may have prevented tissue necrosis in a case of doxorubicin extravasation.

Early surgical consultation is advisable to allow expert assessment of the extent of damage and the need for early surgery. The importance of early excision is stressed by Linder et al., ${ }^{27}$ who noted a higher incidence of residual joint stiffness and sympathetic dystrophy related to prolonged delay in excision or necrotic tissue. Also, adequate splintage and exercises may reduce the risk of contractures in poor functional positions.

Conservative management has been advocated ${ }^{34}$ on the basis that it is difficult to determine the extent of tissue resection and to produce a satisfactory bed for graft or flap take. These views are based on unsuccessful transfer of one flap to what was a probably inadequately excised bed and the conclusions are invalid. Zweig et al. ${ }^{35}$ treated ulcers in 5 patients with a regimen of initial enzymatic debridement followed by $0.5 \%$ sodium hypochlorite solution soaks daily. Sodium hypochlorite was selected on the basis that it depolymerizes DNA and would aid in dissociation of doxorubicin-DNA complexes. However, the onset of
Table II Suggested protocol for immediate management of vesicant extravasation

Aspirate residual drug

No local infusants

Mark area of visible or palpable change with skin marking pen

Colour photograph

Elevate limb

Accurate documentation

Refer to a plastic surgeon the same day

healing was delayed up to 6 weeks, and this delay is probably unacceptable.

In view of the avidity of tissue binding and the progressive nature of tissue necrosis, excision or extravasation of lesions would seem to be logical. Early excision and mesh grafting within 48 hours has been recommended for doxorubicin ${ }^{36}$ extravasation lesions, with an analogy to excisional therapy for pit viper bites. ${ }^{37}$

Excision should include removal of all indurated, reddened, oedematous and pale tissue ${ }^{38}$ probably with a margin of normal appearing tissue. Nonviable doxorubicin-infiltrated tissue can be further identified by reddish orange fluorescence under ultraviolet light in darkness after the intravenous injection of $10 \mathrm{mg} / \mathrm{kg}$ body weight of fluorescein ${ }^{39}$ and by frozen section auto-fluorescence. ${ }^{38}$ This manoeuvre is helpful as skin grafts take poorly in the presence of residual doxorubicin. ${ }^{38}$

Larson, ${ }^{24}$ however, takes a divergent view and recommends delayed excision, only in the presence of persistent local pain or persistent skin changes, believing that thereby less radical excision is possible and unnecessary surgery may be avoided. Staged excisions may be necessary when the completeness of initial excision is uncertain.

The type of wound coverage required is dictated by the nature of the postexcisional recipient bed. Meshed split skin-grafts are suitable in the absence of exposed bare cortical bone, bare tendons and major nerves and blood vessels. In the latter instances flap cover is needed.

In seriously ill patients with limited life expectancy, less than optimal cover may be opted for to allow earlier discharge and more effective palliative care. ${ }^{40}$

\section{Conclusions}

While prevention is vitally important, a more aggressive management of extravasation injuries is recommended in order to minimize morbidity. A high degree of vigilance of ongoing infusions and accurate 
documentation of all extravasations are mandatory. Early excision is advisable in most localized extravasations, especially on the dorsum of the hand, where

\section{References}

1. Taylor, T.H. Pitfalls in invasive vascular procedures. Protection Matters, 1983, No. 5, Medical Protection Society, London.

2. Upton, J., Mulliken, J.B. \& Murray, J.E. Major intravenous extravasation injuries. Am J Surg 1979, 137: 497-506.

3. Wang, J.J., Cortes, E., Sinks, L.F. \& Holland, J.F. Therapeutic effect and toxicity of Adriamycin in patients with neoplastic disease. Cancer 1971, 28: 837-843.

4. Dorr, R.T. \& Fritz, W.L. Cancer Chemotherapy Handbook. Henry Kimpton, London, 1980, Chapter 5, pp. 109-111.

5. Linder, R.M., Upton, J. \& Osteen, R. Management of extensive doxorubicin hydrochloride extravasation injuries. J Hand Surg. 1983, 8: 32-38.

6. Reilly, J.J., Neifeld, J.P. \& Rosenberg, S.A. Clinical course and management of accidental Adriamycin extravasation. Cancer 1977, 40: 2053-2056.

7. Rudolph, R., Stein, R.S. \& Patillo, R. Skin ulcers due to Adriamycin. Cancer 1976, 38, 1087-1094.

8. Luedke, D.W., Kennedy, P.S. \& Rietschel, R.L. Histopatho genesis of skin and subcutaneous injury induced by Adriamycin. Plast Reconstr Surg 1985, 63: 463-465.

9. Rudolph, R., Suzuki, M. \& Luce, J. Experimental skin necrosis produced by Adriamycin. Cancer Treat Rep 1979, 63:529-537.

10. Rudolph, R., Woodward, M. \& Hurn, L. Ultrastructure of doxorubicin (Adriamycin) induced skin necrosis in rats. Cancer Res 1979, 39: 3689-3693.

11. Garnick, M., Israel, M., Khetarpal, V. \& Luce, J. Persistence of anthracycline levels following dermal and subcutaneous adriamycin extravasation. Proc Am Assoc Cancer Res 1981, 4: 96-99.

12. Silvestrini, R., Gambarucci, C. \& Dasdia, T. Attivita biologica dell 'adriamicina in vitro. Tumori 1970, 56: 137-148 (cited by Rudolph, R., Suzuki, M., Luce, J.K. Cancer Treat Rep 1979, 63: 529.

13. Hankin, F.M. \& Louis, D.S. Surgical management of doxorubicin (Adriamycin) extravasation. J Ped Orthop 1984, 4: 96-99.

14. Zweig, J.I., Kabakow, B., Wallach, R.C. Valencic, M. \& Zalusky. Rational effective medical treatment of skin ulcers due to Adriamycin. Cancer Treat Rep 1979, 63: 2101-2103.

15. Coleman III, J.J., Walker, A.P. \& Didolkar, M.S. Treatment of Adriamycin induced skin ulcers. A prospective controlled study. J Surg Oncol 1983, 22: 129-135.

16. Myers, C.E., McGuire, W. \& Young, R. Adriamycin: amelioration of toxicity by alpha-tocopherol. Cancer Treat Rev 1976, 60: 961-962.

17. Reilly, J.J., Neifeld, J.P. \& Rosenberg, S.A. Clinical course and management of accidental Adriamycin these injuries can be considered analogous to thermal injuries. The literature has been reviewed in order to guide the reader. extravasation. Cancer 1977, 40: 2053-2056.

18. Desai, M.H. \& Teres, D. Prevention of doxorubicininduced skin ulcers in rats and pigs with dimethylsulphoxide (DMSO). Cancer Treat Rep 1982, 66: $1371-1374$.

19. Olver, I.N. \& Schwartz, M.A. Use of dimethylsulfoxide in limiting tissue damage caused by extravasation of doxorubicin. Cancer Treat Rep 1983, 67: 407-408.

20. Dorr, R.T. \& Alberts, D.S. Pharmacologic antidotes to experimental doxorubicin skin toxicity: a suggested role for beta-adrenergic compounds. Cancer Treat Rep 1981, 65: $1001-1006$.

21. Petro, J.A., Graham, III, W.P., Miller, S.H., Overholt, T. \& Fallon, T. Experimental and clinical studies of ulcers induced with Adriamycin. Surg Forum 1979, 50: 535-537.

22. Tully, T.L., Friedland, G.H. \& Baldini, L.M. Complications of intravenous therapy with steel needles and teflon catheters. Am J Med 1981, 70: 702-706.

23. Ignoffo, R.J. \& Friedman, M.A. Therapy of local toxicities caused by extravasation of cancer chemotherapeutic agents. Cancer Treat Rev 1980, 7o 17-27.

24. Larson, D.L. What is the appropriate management of tissue extravasation by antitumor agents? Plast Reconst? Surg 1985, 75: 397-402.

25. Barlock, A., Howser, D. \& Hubbard, S. Nursing management of Adriamycin extravasation. $A m \mathrm{~J}$ Nursing 1979, 137: 94-96.

26. Zweig, J.I. \& Kabakow, B. An apparently effective counter-measure for doxorubicin extravasation. $\mathrm{J} \mathrm{Am}$ Med Ass 1978, 239: 2116 (letter).

27. Linder, R.M., Upton, J. \& Osteen, R. Management of extensive doxorubicin hydrochloride extravasation injuries. J Hand Surg 1981, 8: 32-38.

28. Donaldson, S.S., Glick, J.M. \& Wilbury, J.R Adriamycin activating a recall phenomenon after radiation therapy. Ann Intern Med 1974, 81: 407-408.

29. Mamakos, M.S . Recall phenomenon of severe skin and muscle necrosis following Adriamycin extravasation in the hand. Int Surg 1984, 69: 73-74.

30. Dragon, L.H. \& Braine, H.G. Necrosis of the hand after doxorubicin infusion distal to an arteriovenous fistula. Ann Intern Med 1979, 91: 58-59.

31. Vogelzang, N.J. "Adriamycin flare": a skin reaction resembling extravasation. Cancer Treat Rep 1979, 63: 2067-2069.

32. Ostrowski, M.J. An unusual allergic reaction in a vein following intravenous Adriamycin. Clin Oncol 1976, 2: $179-180$.

33. Souhami, Jr, R.L. \& Feld, R. Urticaria following intravenous doxorubicin administration. J Am Med Ass 1978, 240: 1624-1626.

34. Chait, L.A. \& Dinner, M.I. Ulceration caused by cytotoxic drugs. S Afr Med J 1975, 49: 1935-1936. 
35. Zweig, J.I., Kabakow, B., Wallach, R.C., Valencic, M. \& Zalusky, R. Rational effective medical treatment of skin ulcers due to Adriamycin. Cancer Treat Rep 1979, 63: 2102-2103.

36. Loughlin, R.A., Landeen, J.M. \& Habal, M.B. The management of inadvertent subcutaneous Adriamycin infiltration. Plast Reconstr Surg 1979, 137: 408-412.

37. Huang, T.T., Lynch, J.B., Larson, D.L. \& Lewis, J. The use of excisional therapy in the management of snake bite. Ann Surg 1974, 179: 598-607.
38. Bowers, D.G. \& Lynch, J.B. Adriamycin extravasation. Plast Reconstri Surg 1984, 61: 86-92.

39. Cohen, F.J., Manganaro, J. \& Bezozo, R.C. Identification of involved tissue during surgical treatment of doxorubicin-induced extravasation necrosis. J Hand Surg 1983, 8: 43-45.

40. Lynch, D.J., Key, J.C. \& White, IV, R.R. Management and prevention of infiltration and extravasation injury. Surg Clin N Am 1979, 59: 939-949. 